\title{
Capacity-Fairness Performance of an Ad Hoc IEEE 802.11 WLAN with Noncooperative Stations
}

\author{
Jerzy Konorski \\ Gdansk University of Technology \\ ul. Narutowicza 11/12, 80-952 Gdansk, Poland \\ jekon@eti.pg.gda.pl
}

\begin{abstract}
For an ad hoc IEEE 802.11 WLAN we investigate how the stations' incentives to launch a backoff attack i.e., to configure small minimum and maximum CSMA/CA contention windows in pursuit of a larger-than-fair bandwidth share, affect a proposed capacity-fairness index $(C F I)$. We link $C F I$ to the network size, "power awareness," a station's perception of the other stations' susceptibility to incentives, and the way of learning how the other stations perceive the other stations' susceptibility to incentives.
\end{abstract}

\section{Introduction}

Estimated limits of performance of an IEEE 802.11 WLAN [5] become the more realistic, the richer is the assumed network model; most existing estimates account for PHY-layer bandwidth, MAC and TCP overhead, DATA frame size, number of network stations, station mobility, and channel characteristics. We bring into the picture noncooperative behavior in the form of a backoff attack: each station $n$ is free to configure an arbitrary $w_{n}=\left\langle w_{n, \min }, w_{n, \max }\right\rangle$ (the minimum and maximum CSMA/CA contention windows) in pursuit of a larger-than-fair long-term bandwidth share [1], [3], thus engaging in a noncooperative CSMA/CA game [3], [7]. It can be shown that if the greedy $<1,1>$ configuration is ruled out (the backoff mechanism is mandatory) then the game has a unique Nash equilibrium (NE) [4]. Otherwise, one disincentive to configure $<1,1\rangle$ is a certain "power awareness" i.e., fear of another station also configuring $<1,1\rangle$, for all the transmission power is then spent on frame collisions. This we assume tantamount to a "penalty" bandwidth share, which leads to multiple Nash equilibria. In the absence of a compelling unique NE we introduce a simple calculus of backoff attack incentives, a form of seeking a best reply to the beliefs as to the other stations' imminent play. We propose a capacity-fairness index (CFI), a synthetic performance measure equal to the product of the total goodput (bandwidth utilization) and the Jain index of the stations' bandwidth shares. We link CFI to the network size, the stations' "power awareness," a station's perception of the other stations' susceptibility to incentives, and a station's way of learning how the other stations perceive the other stations' susceptibility to incentives. Based on the stations' bandwidth shares $b_{n}$ obtained from existing models [2], [7] we demonstrate that for small enough networks and "power aware" enough stations, cooperative behavior may ultimately emerge. 


\section{CSMA/CA Game and Backoff Attack Incentives}

To reflect both the total bandwidth utilization $\Sigma_{n} b_{n}$ and Jain fairness [6] we use their product i.e., $\left(\Sigma_{n} b_{n}\right)^{3} /\left(N \cdot \Sigma_{n} b_{n}^{2}\right)$, that we name capacity-fairness index $(C F I)$. For an $N$ player CSMA/CA game with payoffs $b_{n}$, suppose that $x$ selfish stations configure $w_{\mathrm{s}}=$ $<2,2>, y$ greedy stations configure $w_{\mathrm{g}}=<1,1>$ (i.e., disengage the backoff scheme), and $N-x-y$ honest ones stick to a standard-prescribed $w_{\mathrm{h}}$ e.g., $<16,1024>$; let the respective payofs be denoted by $b_{\mathrm{s}[\mathrm{g}, \mathrm{h}]}(N, x, y)$. In existing IEEE 802.11 settings, $b_{\mathrm{h}}(N$, $0,0)>0$ for not too large $N$ and $b_{\mathrm{h}}(N, x>0, y) \approx 0$. Thus if $x=y=0$ then $C F I=$ $N \cdot b_{\mathrm{h}}(N, 0,0)$ (denote this cooperative value by c-CFI). Note that $b_{\mathrm{G}}=b_{\mathrm{g}}(N, x, 1)$ is the highest possible payoff, while $b_{\mathrm{s}[\mathrm{h}]}(N, x, y>0)=0$. Let $b_{\mathrm{g}}(N, x, y>1)=b_{\mathrm{C}} \leq 0$, where $b_{\mathrm{C}}$ is a "penalty" payoff, reflecting the fact that a greedy (yet "power aware") station in this case spends all its transmission power to no effect. If $b_{\mathrm{C}}<0\left[b_{\mathrm{C}}=0\right]$ then any configuration profile with $y=1[y>0]$ is a NE. Thus the game has multiple Nash equilibria; to predict its outcome we calculate backoff attack incentives.

Definition 1. A selfish [greedy] backoff attack incentive is the ratio of the likely payoff upon switching from $w_{\mathrm{h}}$ to $w_{\mathrm{s}}\left[w_{\mathrm{g}}\right]$, and the cooperative payoff $b_{\mathrm{h}}(N, 0,0)$.

A 0 -order sophisticated approach to the "likely" part neglects similar conduct at the other stations: $I_{\mathrm{g}, 0}=\hat{b}_{\mathrm{g}}(N, 0,1)$ and $I_{\mathrm{s}, 0}=\hat{b}_{\mathrm{s}}(N, 1,0)$ (the hats normalize w.r.t. $b_{\mathrm{h}}(N$, 0 , 0)). Alternatively, a station forms a model of how the other stations' play is susceptible to the calculated incentives. A susceptibility map $\Phi$ returns for a $\left(I_{\mathrm{s}}, I_{\mathrm{g}}\right)$ pair the probabilities $p_{\mathrm{s}}\left[p_{\mathrm{g}}\right]$ of configuring $w_{\mathrm{s}}\left[w_{\mathrm{g}}\right]$ at any other station $\left(p_{\mathrm{h}}=1-p_{\mathrm{s}}-p_{\mathrm{g}}\right.$ is the probability of staying at $w_{\mathrm{h}}$ ). Intuitively, $\Phi$ should be continuous and ensure that $p_{\mathrm{g}}$ increases in $I_{\mathrm{g}}, p_{\mathrm{s}}$ increases in $I_{\mathrm{s}}$, and $p_{\mathrm{h}}$ decreases in both $I_{\mathrm{s}}$ and $I_{\mathrm{g}}$. Taking $\left(p_{\mathrm{s}}, p_{\mathrm{g}}\right)=$ $\Phi\left(I_{\mathrm{s}, 0}, I_{\mathrm{g}, 0}\right)$, one can calculate the expected normalized payoffs:

$$
I_{\mathrm{s}[\mathrm{g}], 1}=\sum_{\substack{x, y, z \geq 0 \\
x+y+z \leq N-1}}\left(\begin{array}{c}
N-1 \\
x y z
\end{array}\right) p_{\mathrm{s}}^{x} p_{\mathrm{g}}^{y} p_{\mathrm{h}}^{z} \cdot \hat{b}_{\mathrm{s}[\mathrm{g}]}(N, x+1[x], y[y+1]) .
$$

This approach can be termed 1-order sophisticated, as it does account for the other stations also calculating incentive measures, though neglects their use of $\Phi$. Higherorder sophistication consists in re-applying (1) to account for the other stations using $\Phi$, their accounting for the other stations using $\Phi$ etc. In the limit $\Phi$ is deemed common knowledge [4]. Hence, $\infty$-order sophisticated incentive measures solve the fixpoint-type equation (where $F$ is defined by (1) with $\left.\left(p_{\mathrm{s}}, p_{\mathrm{g}}\right)=\Phi\left(I_{\mathrm{s}, \infty}, I_{\mathrm{g}, \infty}\right)\right)$ :

$$
\left(I_{\mathrm{s}, \infty}, I_{\mathrm{g}, \infty}\right)=F\left(I_{\mathrm{s}, \infty}, I_{\mathrm{g}, \infty}\right),
$$

A unique solution of (2) obtains e.g., if $\Phi$ is defined as follows:

$$
p_{\mathrm{s}}=\varphi^{2}\left(I_{\mathrm{s}, \infty}\right) /\left(\varphi\left(I_{\mathrm{s}, \infty}\right)+\varphi\left(I_{\mathrm{g}, \infty}\right)\right), p_{\mathrm{g}}=\varphi^{2}\left(I_{\mathrm{g}, \infty}\right) /\left(\varphi\left(I_{\mathrm{s}, \infty}\right)+\varphi\left(I_{\mathrm{g}, \infty}\right)\right) .
$$

Here, the function $\varphi$ measures a station's willingness to switch from $w_{\mathrm{h}}$ to $w_{\mathrm{s}}\left[w_{\mathrm{g}}\right]$, given $\left(I_{\mathrm{s}}, I_{\mathrm{g}}\right)$; it should be continuous and nondecreasing, with $\varphi(0)=0$ and $\varphi(\infty)=1$.

If the CSMA/CA game is played, we use the expected value of CFI w.r.t. the probabilities of configuring $w_{\mathrm{g}}, w_{\mathrm{s}}$, and $w_{\mathrm{h}}$, determined by $\infty$-order incentives. 
Definition 2. The noncooperative CFI, denoted n-CFI, is defined as

$$
\mathrm{c}-C F I \cdot p_{\mathrm{h}}^{N}+p_{\mathrm{g}}\left(1-p_{\mathrm{g}}\right)^{N-1} b_{\mathrm{G}}+p_{\mathrm{s}} \sum_{x=0}^{N-1}\left(\begin{array}{c}
N-1 \\
x
\end{array}\right) p_{\mathrm{s}}^{x} p_{\mathrm{h}}^{N-1-x}(x+1) b_{\mathrm{s}}(N, x+1,0)
$$

(recall that if all $k$ nonzero payoffs out of $N$ are equal then the Jain index is $k / N$ ).

In reality, the other stations' susceptibility to incentives may be learned by playing the CSMA/CA game repeatedly and observing successive configuration profiles. We model this intuition by taking a sigmoid $\varphi(I)=\left(1-e^{-4 I}\right) /\left(1-e^{-4(I-a)}\right)$ and manipulating its center $a$. In the $i^{\text {th }}$ instance of the game, station $n$ 's perception of the other stations' susceptibility is reflected by $a_{n}^{i}$, with the dynamics $a_{n}^{i+1}=\max \left\{0, a_{n}^{i}+\delta_{n}\left(X^{i}, Y^{i}\right)\right\}$. Here, the function $\delta_{n}$ describes the learning process at station $n$, and $X^{i}\left[Y^{i}\right]$ is the number of selfish [greedy] stations in the $i^{\text {th }}$ instance, distributed according to $p_{\mathrm{s}}, p_{\mathrm{g}}$, and $p_{\mathrm{h}}=1-p_{\mathrm{s}}-p_{\mathrm{g}}$ as calculated from (3), with the sigmoid $\varphi$ centered at $a_{n}^{i}$.

Let $\delta_{n}(x>0,0)=-\Delta_{n}, \delta(0,0)=-2 \Delta_{n}$, and $\delta(x, y>0)=\Delta_{n}$, where $\Delta_{n}$ is proportional to station $n$ 's initial $a_{n}^{0}$ through a constant $\Delta$ (thus relative changes of $a$ are the same at each station). $\left(\left(a_{1}^{i}, \ldots, a_{N}^{i}\right), i=1,2, \ldots\right)$ is an $N$-dimensional random walk with an absorbing state $(0, \ldots, 0)$ corresponding to cooperative behavior (the solution of (2) then yields $\left.p_{\mathrm{s}}=p_{\mathrm{g}}=0\right)$ and another absorbing state $(\infty, \ldots, \infty)$, with $p_{\mathrm{s}}=p_{\mathrm{g}}=1 / 2$ and $p_{\mathrm{h}}$ $=0$. The capacity-fairness indices at these absorbing states are c-CFI and $\mathrm{n}-C F I_{\infty}=$ $\left(N \cdot b_{\mathrm{s}}(N, N, 0)+b_{\mathrm{G}}\right) / 2^{N}$, respectively. Define $a_{\max }$ so that the corresponding solution of (2) yields $p_{\mathrm{s}}$ and $p_{\mathrm{g}}$ close to $1 / 2$.

Definition 3. The noncooperative learning CFI, denoted nl-CFI, is defined as $\pi_{N} \cdot \mathrm{c}-$ $C F I+\left(1-\pi_{N}\right) \cdot \mathrm{n}-C F I_{\infty}, \pi_{N}$ being the probability of reaching the absorbing state $(0, \ldots, 0)$ given that each station $n$ selects $a_{n}^{0} \in\left[0 \ldots a_{\max }\right]$ at random.

Assuming $\Delta=0.2$, Fig. 1 depicts $\mathrm{c}-C F I$ and nl-CFI. The latter turns out to be distinctly closer to c-CFI than n-CFI for "power aware" enough stations, as confirmed by numerical experiments whose details are omitted.

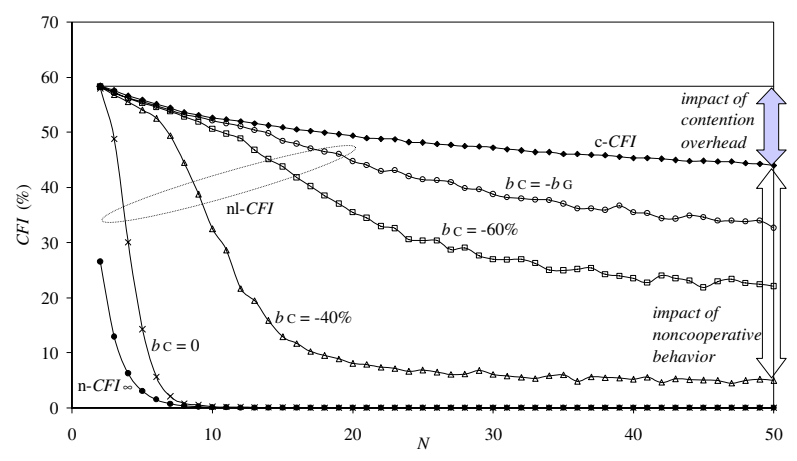

Fig. 1. c-CFI and nl-CFI for various "power awareness" levels 


\section{Conclusion}

The introduction of $w_{\mathrm{g}}$ and "power awareness" changes the CSMA/CA game into one with multiple Nash equilibria i.e., without a compelling outcome. We envisage that each station then calculates common-knowledge incentives to configure $w_{\mathrm{s}}$ and $w_{\mathrm{g}}$, and the corresponding probability distribution of imminent configuration profiles. Our study quantitatively illustrates a few intuitions:

- the network's ability to provide high and fair bandwidth shares to all stations diminishes as $N$ increases, partly on account of growing contention overhead, but mostly because of the stations' limited willingness to behave cooperatively; these two factors are illustrated for the $b_{\mathrm{C}}=-40 \%$ curve at $N=50$,

- incentive calculus dictates that the willingness to behave cooperatively grow with "power awareness" for fear of spending all the transmission power without getting any bandwidth share; accordingly, $C F I$ improves as $b_{\mathrm{C}}$ goes more negative,

- the predictions depend on a station's perception of the other stations' susceptibility to incentives, reflected by $\Phi$, and the learning process, reflected by $\delta$,

- each of the nl-CFI curves lies between the n-CFI $I_{\infty}$ and c-CFI ones; its bias towards the latter measures the chance $\pi_{N}$ of emergence of cooperative behavior; this is almost certain for small enough $N$ assuming enough "power awareness."

Although the "penalty" bandwidth share $b_{\mathrm{C}}$ was assumed constant across the stations, it is relatively easy to generalize to nonuniform "power awareness" in order to study the coexistence of devices with diverse battery lifetimes.

\section{Acknowledgment}

This work was supported by the Ministry of Education and Science, Poland, under Grant 1599/T11/ 2005/29.

\section{References}

1. Bellardo, J., Savage, S.: 802.11 Denial-of-Service Attacks: Real Vulnerabilities and Practical Solutions, Proc. USENIX Security Symp., Washington DC (2003)

2. Bianchi, G.: Performance Analysis of the IEEE 802.11 Distributed Coordination Function, IEEE J. on Selected Areas in Comm. 18 (2000) 535-547

3. Cagalj, M., Ganeriwal, S., Aad, I., Hubaux, J.-P.: On Cheating in CSMA/CA Ad Hoc Networks, Proc. IEEE INFOCOM 2005, Miami FL (2005)

4. Fudenberg, D., Tirole, J.: Game Theory. MIT Press (1991)

5. IEEE Standard for Information Technology: LAN/MAN - Specific Requirements, ISO/IEC 8802-11 (1999)

6. Jain, R.: Fairness: How to Measure Quantitatively? ATM Forum/94-0881 (1994)

7. Konorski, J.: A Game-Theoretic Study of CSMA/CA Under a Backoff Attack, IEEE/ACM Trans. on Networking, 14 (2006) 1167-1178 